\title{
Triangle and Trapezoid Area Features for Gait Authentication
}

\author{
Tasriva Sikandar, Wan Nur Azhani \\ W Samsudin \\ Faculty of Electrical and Electronics \\ Engineering \\ Unviersiti Malaysia Pahang \\ Pekan 26600, Malaysia \\ tasrivasikandar@gmail.com
}

\author{
Kamarul Hawari Ghazali, Mohammad \\ Fazle Rabbi \\ Faculty of Electrical and Electronics \\ Engineering \\ Unviersiti Malaysia Pahang \\ Pekan 26600, Malaysia
}

\begin{abstract}
This paper presents two gait authentication features based on geometric shape for gait analysis. Specifically, triangle and trapezoid based features are proposed for gait authentication. The features are based on the geometric pattern extracted from a particular gait cycle of a gait model. These features use four points from hip-knee-toe joints and construct a triangle and a right trapezoid. The area of the triangle and trapezoid are calculated using geometric formula as well as image processing methods. Later two areas are compared to validate the model free approach. The results show that, the proposed feature can be used as the features in model free gait analysis.
\end{abstract}

Keywords—gait; feature; triangle; trapezoid; authentication

\section{INTRODUCTION}

Image based human gait authentication or recognition has become an important research area due to the increasing demand of security issue as well as huge potential of medical applications for abnormal gait detection. For example, vision based gait recognition can be a useful tool in forensic analysis of crime where no fingerprint is available [1], [2]. In addition, gait recognition has become one of the most reliable applications in the area of physical therapy, biometrics, rehabilitation, sports, science, and geriatric care [3]-[5]. Clinicians are also able to utilize gait segmentation concept in their routine clinical practice to evaluate a patient's status, treatment, and rehabilitation for complex musculoskeletal and neurological disorders [2], [6]. In the gait authentication system, a person is identified from the pattern of walking which is an outcome of a synchronized cycle of leg movement. Usually, gait recognition methods can be classified into two types based on the methodology; $i$ ) model based and ii) holistic or model free [7]. In addition, the methods require extraction of a unique feature from recorded image/video to draw a conclusion for the identification [8].

In the model based methods, the body configuration is captured in motion. The features extracted during the motion are modelled and matched to the designed model features [2]. The knowledge of the body shape and the kinematic gait features are combined. The main advantage of the modelbased approach is that it can reliably handle occlusion (especially self-occlusion), noise, scale and rotation well, as opposed to silhouette-based approaches [9]. However, this method produces many parameters from extracted features and thus, results in a complex model to be analysed. On the other hand, model free gait recognition method is an appearance based method which works directly on the gait sequences [10], [11]. This does not consider a model for the human body to rebuild human walking steps. They have the advantage of low computational cost in comparison with model-based approaches. Instead, its parameters are obtained from the static gait features like centroid, width and height of the silhouette.

Among the holistic approaches, two types of methods are used frequently; $i$ ) frame based and ii) gait cycle based [10]. In frame based technique, features are extracted from each frame of the video sequence, while in gait cycle based approach, features are extracted from a single image calculated over a gait cycle. Gait energy [12], entropy [13], flow [14] and probability [15] are examples of gait cycle based techniques. Angles between hip-knee and knee-toe joints were studied as gait features in [16]. Also, [17] investigated some more gait features such as adaptive height, neck joint, hip and knee joints. In addition, previously used models include stick and volumetric models, which are most commonly used [18]. In this method a model consisting of six segments comprising of two arms, two legs, torso and head represent the human body structure in the silhouette by a stick figure model consisting ten sticks articulated with six joints. Due to the higher advantages and better results, [17], [19] have combined model based and holistic approach to extract the subsequent gait features. Model free features in those studies include adaptive height, neck joint, hip and knee joints. In addition, model based features were the area under the legs during the gait cycle and Beizer and hermite implementation of the extracted joints. However, due to the parametric analysis this method may also suffer from high computational complexity. Therefore, finding a simple but efficient feature for gait cycle authentication is always a challenging issue.

In this paper, we propose two unique geometrical shapes during gait cycle so that, the area of the shapes can be used as a gait feature. Specifically, a triangle and a right trapezoid, between two legs are identified in cyclic manner during a normal gait cycle. We use four points from the silhouette of a gait model such as, hip joint, knee joint, toe and ground. Based 
on the points a triangle can be identified in around $0 \%, 50 \%$ and $100 \%$ of a gait cycle. Also, a right trapezoid can be specified in around $39 \%$ and $90 \%$ of the cycle. The present study proposes that the triangle and trapezoid shape area can be used as a unique gait feature. To validate the reliability of these proposed gait features, we have applied mathematical formula incorporate with image processing method. In addition, validation of the proposed features has been demonstrated in terms of correlation analysis and average area comparison.

The rest of the article is organized as follows. Section II presents proposed theory of triangle and trapezoid area based features. Description of validation process and experiment including data acquisition is given in Section III and IV respectively. Results and discussion on validation result is presented in Section V. Finally, some concluding remarks have been given in Section VI.

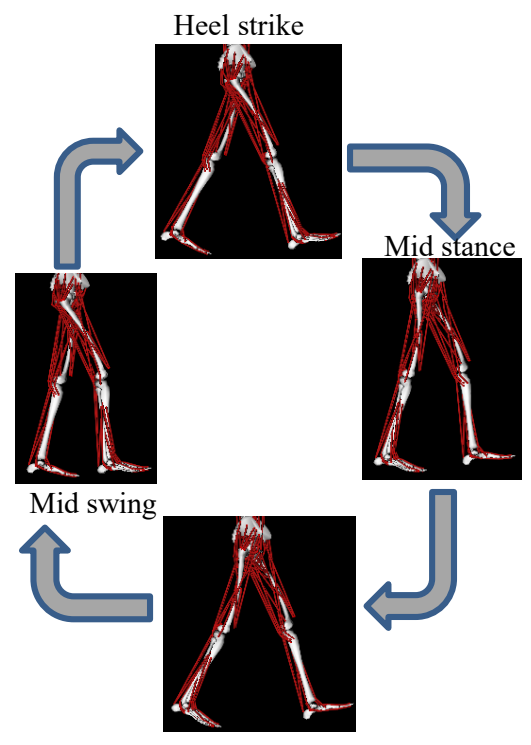

Fig.1. Repetition of heel strike, mid stance and mid swing in a cycle

\section{PROPOSED METHOD}

Five sub-phases in a complete gait cycle are known as heel strike, mid stance, opposite heel strike, mid swing and end of cycle. The sub-phases are repeated in each gait cycle as shown in Fig. 1. In addition, by evaluating four points from a gait model such as, hip joint, knee joint, toe and ground, a triangular shaped area can be observed between two legs during $0 \%, 50 \%$ and $100 \%$ cycle. Furthermore, a right trapezoid shaped area can be noticed in $40 \%$ and $90 \%$ of the gait cycle. This implies that, the repetition of a triangular shape and a right trapezoid shape appears thrice and twice from heel strike to opposite heel strike in a gait cycle. Fig. 2 presents complete gait cycle of a normal gait model while Fig. 3 shows trace of a triangle at $0 \%, 50$ and $100 \%$ and trace of a right-trapezoid during $39 \%$ and $90 \%$ gait cycle. As demonstrated in Fig. 2, other shapes between legs are not as consistent as the triangle and right-trapezoid. Therefore, it is justifiable to use those to geometric shape as features of normal gait authentication. Note that, the OpenSim gait model,

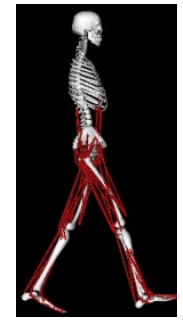

$0 \%$

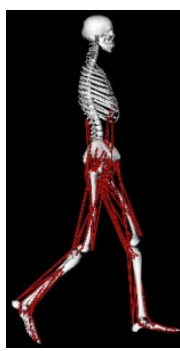

$60 \%$

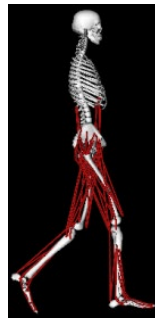

$10 \%$

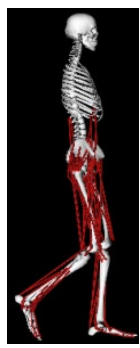

$70 \%$

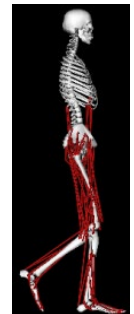

$20 \%$

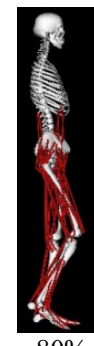

$80 \%$

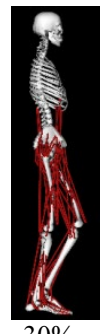

$30 \%$

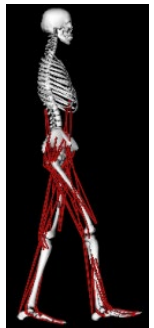

$90 \%$

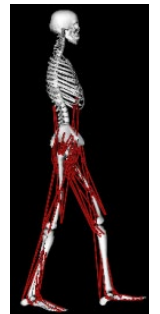

$40 \%$

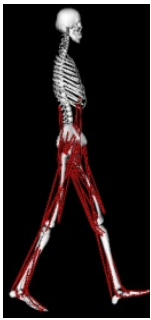

$50 \%$
Fig. 2. Complete gait cycle of a normal gait model in OpenSim. Gait sequence recorded in every $10 \%$ cycle duration

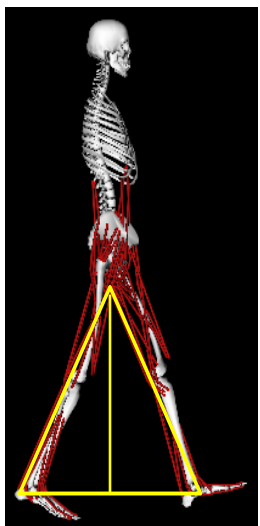

(a)

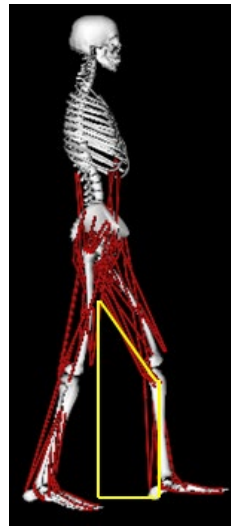

(b)
Fig. 3. Trace of a (a) triangle at $0 \%, 50 \%$ and $100 \%$, (b) right-trapezoid during $39 \%$ and $90 \%$ gait cycle.

developed by [20], has been used to get the images (Fig. 1-3) of a model in normal gait condition. This open source 3D musculoskeletal simulation platform has been used by many recent studies including [21], [22].

With this consideration, the area of the shapes can be used as a gait feature. For the shape area calculation, Fig. 4 presents the schematic stick diagram of the gait model. As depicted in the Fig. 4(a), the height of the triangle is the length between hip and ground while the width is equal to the length between the toe and the hip-ground line.

Therefore, the area of the triangle can be calculated as

$$
A_{\text {triangle }}=\frac{1}{2} h d
$$

where $h$ is the length from hip to ground, $1 / 2 d$ is the length between mid-ground point to toe. Similarly, Fig. 3(b) presents the right trapezoid between two legs where the height and 


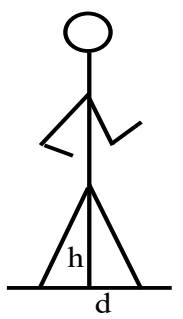

(a)

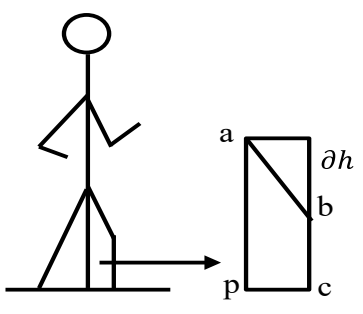

(b)
Fig. 4 Area calculations of (a) triangle (b) right trapezoid from stick schematic representation of gait model

width are $h$ and $1 / 2 d$ respectively. The area of the right trapezoid can be calculated as

$$
A_{\text {trapezoid }}=h d-\frac{1}{4}(\partial h) d
$$

where $\partial h=\sqrt{a b^{2}-(0.25 d)^{2}}=h-b c$ and $a b$ is the length between hip and knee, $a p=h$ and, $b c$ is the length between knee and ground.

\section{VALIDATION METHOD}

To validate the triangle and trapezoid shape area as unique gait features, we have employed both geometric formula as well as image processing methods. Specifically, the region in between legs will be extracted first from the gait model image. Next, we calculate the area in-between two legs using two different processes. In the shape based calculation (SBC) the area in-between two legs will be determined by using geometrical formulations of triangle and trapezoid while white pixel count (WPC) based area calculation will give us an estimate of actual area in-between two legs. By doing so we will compare the areas measured in two different methods to see how close the calculated shape based area to the actual area for validation of Triangle and Trapezoid Area based features.

Fig. 5 presents process flow of validation method for triangle and trapezoid area based features. As illustrated in the figure input RGB images of gait model are obtained from OpenSim. We convert the color space of input image from RGB to YCbCr. We detect the red color muscle of the gait model by defining the $\mathrm{Cb}, \mathrm{Cr}$ threshold value range for red color. The red color muscle region is extracted in the form of a binary image, where the muscle region is presented as white pixel. We remove noises from the extracted muscle region. We apply dilation on the extracted image with a horizontal line structuring element. We also apply morphological closing operation on the dilated image to fill in the largest gap inside the extracted muscle region. Specifically, we connected the toe point by inserting a line and then used a square shaped structuring element for morphological closing. We extract the region in-between two legs by subtracting the dilated toe line drawn image from morphologically closed image. We also remove the unconnected extra objects from the resultant image. We calculate the actual area in-between two legs by applying WPC on the extracted area.
Next, we estimate the area in-between two legs using SBC. Specifically, we determine three corner points and middle point of the base of a triangle in the extracted region inbetween two legs for the cases of $0 \%, 50 \%$ and $100 \%$ cycle. We calculate length of the arms of triangle, i.e. height $h$ and base $d$, by applying Euclidean distance (ED) on the extracted points. With the determined height and base, we calculate area of triangle using triangle area formula given in (1). On the other hand, we determine four corner points in the extracted region in-between two legs for the cases of $40 \%$ and $90 \%$ cycle. We calculate length of trapezoid arms, i.e. height $h$, width $d$, length between hip and knee $a b$ and length between knee and ground $b c$, by applying ED on the extracted points. With the determined length of right trapezoid arms, we calculate area of the trapezoid using trapezoid area formula given in (2).

Finally we perform correlation analysis on the areas inbetween two legs measured in two different methods, i.e. WPC and SBC, to see how close the shape based calculated area (i.e. $\mathrm{SBC}$ ) to the actual area (i.e. WPC).

TABLE I. SUMMARY OF DATASET

\begin{tabular}{|l|c|l|c|}
\hline Gait cycle & Sub-phase & Geometric shape & $\begin{array}{c}\text { No of } \\
\text { images }\end{array}$ \\
\hline $0 \%-3 \%,$, & HS & Triangle & 17 \\
\hline $37 \%-41 \%$ & MS & Trapezoid & 17 \\
\hline $47 \%-51 \%$, & OS & Triangle & 17 \\
\hline $87 \%-91 \%$ & MW & Trapezoid & 17 \\
\hline $96 \%-100 \%$ & EC & Triangle & 17 \\
\hline \multicolumn{3}{|c|}{ Total images } & 85 \\
\hline
\end{tabular}

\section{A. Dataset}

\section{EXPERIMENT}

The OpenSim gait model is considered as subjects. We captured images in stance and swing phases of a cycle simulated by 3 DGaitModel2392 in normal gait condition. The walking speed of the model was $1 \mathrm{~m} / \mathrm{sec}$. Images were captured in $0 \%-3 \%, 37 \%-41 \%, 47 \%-51 \%, 87 \%-91 \%$ and $96 \%-100 \%$ for five sub-phases of gait cycle. Note that, these five sub-phases will be referred to as, heel strike (HS), mid stance (MS), opposite heel strike (OS), mid swing (MW) and end of cycle (EC) respectively. 17 images were captured in each sub-phase with a gap of $0.3 \%$ cycle. For example, in the first sub-phase, 17 consecutive images were captured in $0 \%$, $0.3 \%, 0.9 \%$ and so on. Therefore, there are total 85 images which were analysed. The summary of our gait model image dataset has been given in TABLE I. All the images were processed and analysed using MATLABR2014a software in an Intel(R) Core(TM)i5-2400CPU, 3.10GHz computer.

\section{B. Experiment demonstration}

The process of triangle and right-trapezoid area calculation comprises of WPC and SBC calculation. Fig. 6 and Fig. 7 represent the area calculation process of triangle and trapezoid respectively. The area calculation is according to the process demonstrated in Fig. 5. A message box is used to show the 

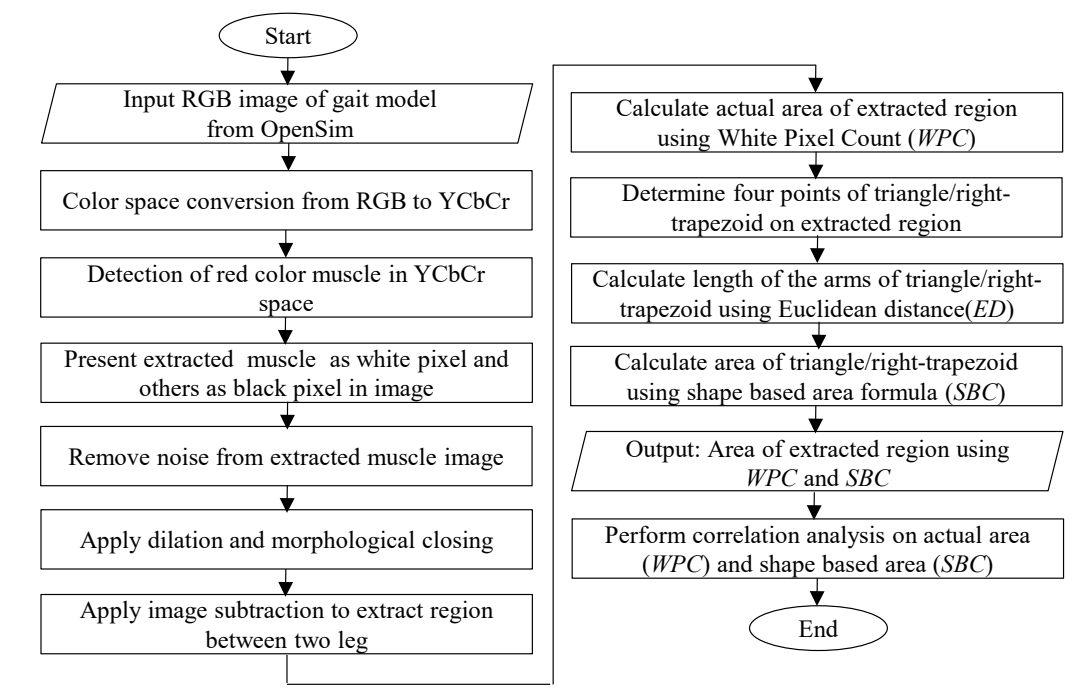

Fig. 5. Process flow of validation method for triangle and trapezoid area based feature extraction

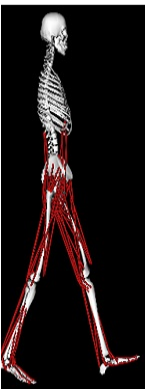

(a)

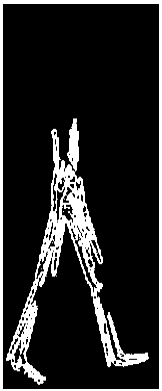

(b)

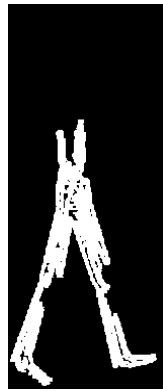

(c)

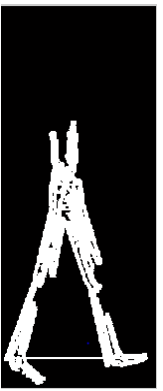

(d)

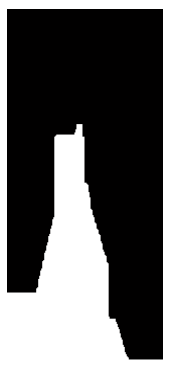

(e)

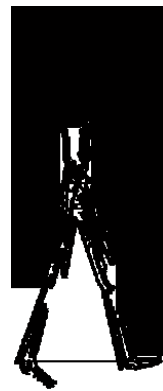

(f)

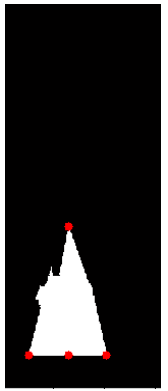

(g)

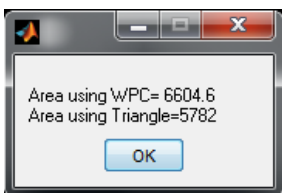

(h)

Fig. 6. Area calculation process using WPC and SBC for triangle observed in $0 \%, 50 \%$ and $100 \%$ cycle (a) RGB input image of gait model (b) extracted muscle using color information (c) dilation of extracted region (d) insertion of toe line (e) morphological closing (f) image subtraction (g) extracted region in-between two legs and the determined three corner points and middle point of base of triangle in the extracted region (h) result: calculated area using WPC and SBC.

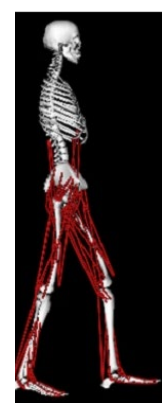

(a)

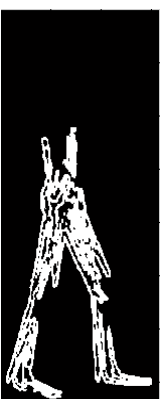

(b)

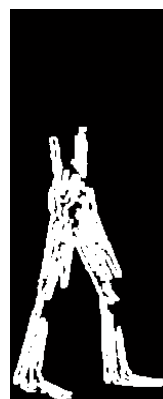

(c)

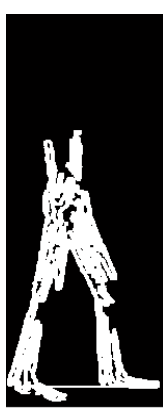

(d)

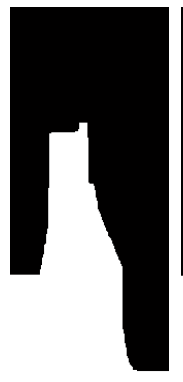

(e)

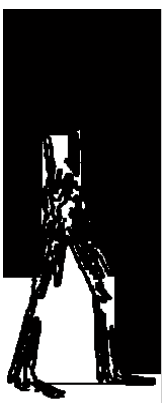

(f)

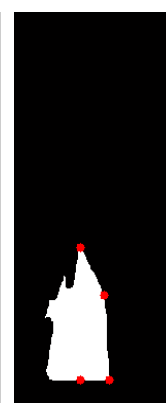

(g)

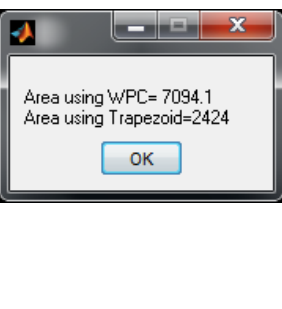

(h)

Fig. 7 Area calculation process using WPC and SBC for right trapezoid observed in 40\% and 90\% cycle (a) RGB input image of gait model (b) extracted muscle region using color information (c) dilation of extracted region (d) insertion of toe line (e) morphological closing (f) image subtraction (g) extracted region inbetween two legs and the determined four corner points of trapezoid in the extracted region (i) result: calculated area using WPC and SBC.

calculated area using WPC and SBC. Note that, the unit of area has not been used similar to [19]. As demonstrated in the figures, the trapezoid area extracted from the gait image is smaller than triangle area. A correlation analysis between two calculated areas will be given in Section-V.

\section{RESULTS AND DISCUSSION}

Fig. 8 presents the average area in-between two legs measure by SBC (triangular formulation) and WPC during HS (0\%-3\%), OS (47\%-51\%) and EC (96\%-100\%). The standard error has also been shown in the figure. As demonstrated in 


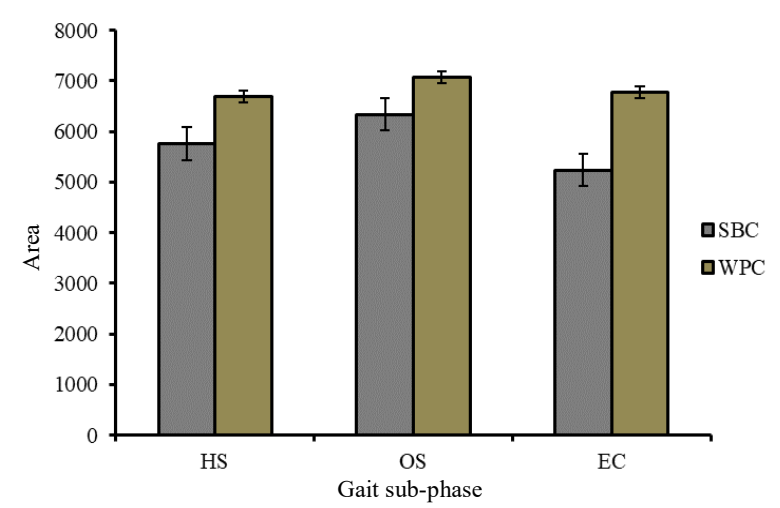

Fig. 8 Average triangle area calculated by SBC and WPC

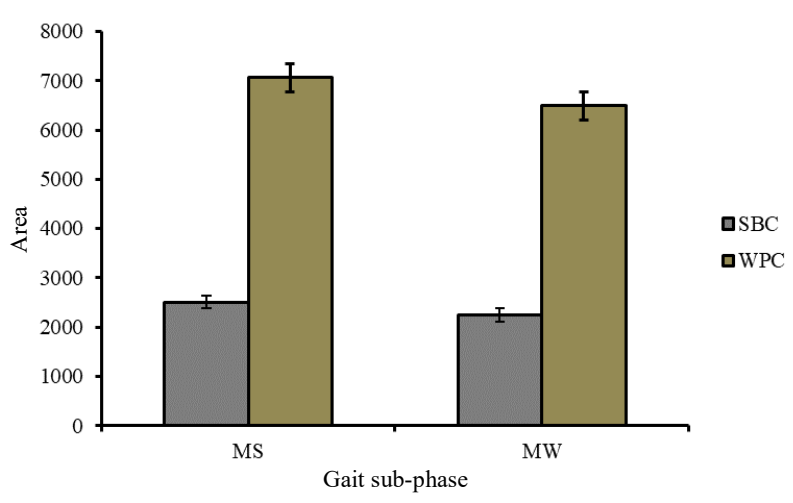

Fig. 9 Average trapezoid area calculated by SBC and WPC

the figure, the calculated area was found almost same for three sub-phases using SBC method. Similar trend was observed in the calculated area using WPC method. This implies that, the area in-between two legs in these three sub-phases are almost similar. In addition, high correlation between the areas was found for the SBC and WPC in all three times. Specifically, $(r=0.973, p<0.05),(r=0.965, p<0.05)$ and $(r=0.866, p<0.05)$ were found in HS, OS and EC respectively. This implies that, the triangular area calculated using geometrical formula is very close to the actual area in-between two legs during those three positions.

Fig. 9 presents the average area in-between two legs measured by SBC and WPC during MS (37\%-41\%) and MW (87\%-91\%). The standard error has also been shown in the figure. As demonstrated in the figure, the calculated area was found almost same for two sub-phases using SBC and WPC method. This implies that, the area in-between two legs in these two sub-phases are almost similar. In addition, the area calculated using WBC is significantly higher than that calculated using SBC. The reason may be right trapezoidal formulation considers only a partial extracted region inbetween two legs. However, the calculated area using SBC was found highly correlated with that using WPC in both times. Specifically, $(r=0.951, p<0.05)$ and $(r=0.956, p<0.05)$ were found in MS and MW respectively. This implies that, the trapezoidal area calculated using geometrical formula is very close to the actual area in-between two legs during those two phases of a gait cycle.
TABLE II. CORRELATION BETWEEN SBC AND WPC CALCULATED TRIANGLE AREA IN THREE SUB-PHASES OF A GAIT

\begin{tabular}{|c|c|c|c|}
\hline SBC/WPC & HS & OS & EC \\
\hline HS & 0.973 & 0.928 & 0.955 \\
\hline OS & 0.97 & 0.865 & 0.962 \\
\hline EC & 0.97 & 0.943 & 0.955 \\
\hline
\end{tabular}

TABLE III. CORRELATION BETWEEN SBC AND WPC CALCULATED TRAPEZOID AREA IN TWO SUB-PHASES OF A GAIT

\begin{tabular}{|c|c|c|}
\hline SBC/WPC & MS & MW \\
\hline MS & 0.964 & 0.972 \\
\hline MW & 0.894 & 0.95 \\
\hline
\end{tabular}

TABLE-II and TABLE-III present the correlation between the triangular and trapezoidal areas calculated using SBC and WPC. As demonstrated in the TABLE-II, the correlation among the consecutive area in HS, OS and EC has been found quite high for calculated area in two methods. Specifically, calculated areas for HS showed the similar correlation with OS $(97 \%)$ and EC (97\%) while the area calculated for OS demonstrated lower correlation with other two phases $(92.8 \%$ and $94.3 \%$ ) including lowest $(86.5 \%)$ for OS area calculation using two methods. In addition, as depicted in TABLE-III, the correlation between the area calculated for MS and MW was found very high as well when SBC and WPC methods were applied. However, the correlation between the areas calculated trapezoidal area for MS was found lower than that of MW. The correlation of MW area calculated by the two methods was found as high as $95.8 \%$ and $95.5 \%$ for MS and MW respectively.

Despite having some significant results, there are few limitations in this study as well. For example, the gait phases were extracted from the captured images of a simulated gait model. The human subjects would have been more appropriate for the study. Nevertheless, since we rely on the image processing method, the images are transformed into silhouette which would be similar regardless the type of image of subjects. Human subjects as well as existing database will be considered in future work. In addition, this work only considers the normal gait while the analysis of abnormal gait will be considered as future work.

\section{CONCLUSION}

In this paper we propose two unique features to identify gait behaviour. Two geometric shapes based on triangle and right trapezoid have been employed to create the features. The area calculated by geometric formula has been validated by the area measured by white pixel count method. High correlation was found between the areas calculated using SBC and WPC method. Specifically, the triangular area calculated in HS, OS and EC sub-phases were found very close to each other while using SBC and WPC method. Similarly, the trapezoidal area calculated during MS and MW was found almost same while using SBC and WPC method separately. The high correlation between the areas calculated in two 
methods implies that, the geometric shape based features can be used as a feature in normal gait authentication.

\section{ACKNOWLEDGEMENT}

This research was supported by the Research \& Innovation Department, University Malaysia Pahang (UMP) Research Grant (No. RDU1703256).

\section{REFERENCES}

[1] S. M. M. De Rossi et al., "Gait Segmentation Using Bipedal Foot Pressure Patterns," in The Fourth IEEE RAS/EMBS International Conference on Biomedical Robotics and Biomechatronics, 2012, pp. 361-366.

[2] C. Prakash, K. Gupta, R. Kumar, and N. Mittal, "Fuzzy LogicBased Gait Phase Detection Using Passive Markers," in Proceedings of Fifth International Conference on Soft Computing for Problem Solving, Advances in Intelligent Systems and Computing, 2016, vol. 437, pp. 561-572.

[3] C. M. Senanayake and S. M. N. Arosha Senanayake, "Evaluation of gait parameters for gait phase detection during walking," IEEE Int. Conf. Multisens. Fusion Integr. Intell. Syst., pp. 127-132, 2010.

[4] H. Wang, J. Wu, Y. Wang, L. Ren, D. Zhang, and H. Lu, "Research on the lower limb gait rehabilitation," 2014 IEEE Int. Conf. Mechatronics Autom. IEEE ICMA 2014, pp. 1243-1247, 2014.

[5] J. Courtney and A. M. De Paor, "A monocular marker-free gait measurement system," IEEE Trans. Neural Syst. Rehabil. Eng., vol. 18, no. 4, pp. 453-460, 2010.

[6] C. S. Yeh, Y. Lin, and Y. Wu, "OFDM system channel estimation using time-domain training sequence for mobile reception of digital terrestrial broadcasting," IEEE Trans. Broadcast., vol. 46, no. 3, pp. 215-220, 2000.

[7] V. Boulgouris, D. Hatzinakos, and K. N. Plataniotis, "Recognition : A challening signal," IEEE Signal Process. Mag., no. November, pp. 78-90, 2005.

[8] M. R. Dawson, "Gait Recognition," 2002.

[9] R. L. Attwells, S. A. Birrell, R. H. Hooper, and N. J. Mansfield, "Influence of carrying heavy loads on soldiers' posture, movements and gait," Ergonomics, vol. 49, no. 14, pp. 1527-1537, 2006.
[10] P. Arora and S. Srivastava, "Gait Recognition using Gait Energy Image," in 2015 2nd International Conference on Signal Processing and Integrated Networks (SPIN), 2015, vol. 4, no. 3, pp. 316-322.

[11] P. Arora, M. Hanmandlu, and S. Srivastava, "Gait based authentication using gait information image features," Pattern Recognit. Lett., vol. 68, pp. 336-342, 2015.

[12] J. Han and B. Bhanu, "Gait Recognition using Gait Energy Image," IEEE Trans. Pattern Anal. Mach. Intell., vol. 4, no. 3, pp. 316-322, 2011.

[13] K. Bashir, T. Xiang, and S. Gong, "Gait Recognition Using Gait Entropy Image," in 3rd International Conference on Imaging for Crime Detection and Prevention, 2009, pp. 1-6.

[14] T. H. W. Lam, K. H. Cheung, and J. N. K. Liu, "Gait flow image: A silhouette-based gait representation for human identification," Pattern Recognit., vol. 44, no. 4, pp. 973-987, 2011.

[15] C. P. Lee, A. W. C. Tan, and S. C. Tan, "Gait probability image: An information-theoretic model of gait representation," J. Vis. Commun. Image Represent., vol. 25, no. 6, pp. 1489-1492, 2014.

[16] J. H. Yoo, M. S. Nixon, and C. J. Harris, "Extracting human gait signatures by body segment properties," Proc. IEEE Southwest Symp. Image Anal. Interpret., vol. 2002-Janua, pp. 35-39, 2002.

[17] P. Arora, S. Srivastava, R. Jain, and P. Tomar, "Parametric Curve Based Human Gait Recognition," Inf. Syst. Des. Intell. Appl., vol. 435, pp. 367-375, 2016.

[18] J. E. Boyd and J. J. Little, Biometric Gait Recognition. Springer Berlin Heidelberg., 2005.

[19] J. P. Foster, M. S. Nixon, and A. Prudel-Bennett, "New Area Based Metrics for Automatic Gait Recognition," in International Conference on Audio-and Video-Based Biometric Person Authentication, 2001, pp. 312-318.

[20] S. L. Delp et al., "OpenSim: Open source to create and analyze dynamic simulations of movement," IEEE Trans. Biomed. Eng., vol. 54, no. 11, pp. 1940-1950, 2007.

[21] H. Xu, D. Bloswick, and A. Merryweather, “An improved OpenSim gait model with multiple degrees of freedom knee joint and knee ligaments," Comput. Methods Biomech. Biomed. Engin., vol. 18, no. 11, pp. 1217-1224, 2015.

[22] Y. C. Lin and M. G. Pandy, "Three-dimensional data-tracking dynamic optimization simulations of human locomotion generated by direct collocation," J. Biomech., vol. 59, pp. 1-8, 2017. 